\title{
Placenta percreta - an audacious experience
}

\section{Shrinivas Gadappa*, Sonali Deshpande, Kalyani Rajpurohit, Bhakti Kalyankar}

Department of Obstetrics and Gynecology, Government Medical College, Aurangabad, Maharashtra, India

Received: 23 July 2016

Accepted: 16 August 2016

\section{*Correspondence:}

Dr. Shrinivas Gadappa,

E-mail: gadappashrinivas@gmail.com

Copyright: ( $)$ the author(s), publisher and licensee Medip Academy. This is an open-access article distributed under the terms of the Creative Commons Attribution Non-Commercial License, which permits unrestricted non-commercial use, distribution, and reproduction in any medium, provided the original work is properly cited.

\begin{abstract}
Maternal and fetal morbidity and mortality from placenta accreta are considerable and are associated with high demands on health resources. There is abnormally firm attachment of placenta to the uterine wall with the absence of deciduas basalis and incomplete development of Nitabuch's layer. The reported incidence of placenta accreta has increased from approximately $0.8 \%$ in 1980 s to 3 per 1000 in the last decade, occurring more frequently in future deliveries after caesarean section. Management of patients with a morbidly adherent placenta (placenta accreta, increta, or percreta) varies widely. Although the impact of a morbidly adherent placenta on pregnancy outcomes is well-described, no randomized trials have examined the management of pregnancies complicated by this disorder. As a result, recommendations for its management are based on case series and reports, personal experience, expert opinion, and good clinical judgement. We report a case of placenta percreta that was successfully managed by planned caesarean hysterectomy with prophylactic ligation of bilateral anterior division of internal iliac artery.
\end{abstract}

Keywords: Placenta accreta/percreta, Massive transfusion protocol, Internal iliac artery ligation

\section{INTRODUCTION}

Maternal and fetal morbidity and mortality from placenta accreta are considerable and are associated with high demands on health resources. There is abnormally firm attachment of placenta to the uterine wall with the absence of deciduas basalis and incomplete development of Nitabuch's layer. ${ }^{1}$ The reported incidence of placenta accreta has increased from approximately $0.8 \%$ in $1980 \mathrm{~s}$ to 3 per 1000 in the last decade, occurring more frequently in future deliveries after caesarean section. ${ }^{2}$ Management of patients with a morbidly adherent placenta (placenta accreta, increta, or percreta) varies widely. Although the impact of a morbidly adherent placenta on pregnancy outcomes is well-described, no randomized trials have examined the management of pregnancies complicated by this disorder. As a result, recommendations for its management are based on case series and reports, personal experience, expert opinion, and good clinical judgement. Here we report a case of placenta percreta that was successfully managed by planned caesarean hysterectomy with prophylactic ligation of bilateral anterior division of internal iliac artery.

\section{CASE REPORT}

A 28 year old woman, G4P2L2A1 with previous two caesarean deliveries referred from a private hospital to Government Medical College, Aurangabad with 3 months of amenorrhoea and ultrasound report suggestive of Placenta accreta.

On examination, her vitals were stable, per abdomen examination revealed uterus of 12-14 weeks of gestational age. Per vaginal examination was not done. $\mathrm{Her} \mathrm{Hb}$ was $10 \mathrm{gm} \%$.

Her ultrasound at 14 weeks of gestation was suggestive of grade 4 placenta previa with vascularity reaching anteriorly in myometrium possibly increta and repeat scan at 20 weeks indicated grade 4 placenta previa with myometrial thinning, placental lakes and placental vessels reaching up to serosa suggestive of placenta 
percreta. MRI scan was done and was consistent with USG findings.

A proposition for continuing the pregnancy till 34 completed weeks of gestation followed by planned caesarean delivery with obstetric hysterectomy was taken. She and her relatives were explained about the risk and prognosis of continuation of such pregnancy. She was made cognizant of the warning symptoms i.e. pv bleeding, acute pain in abdomen and explained the need to report immediately if the same occurs. Meanwhile she had regular antenatal check-up at GMC, Aurangabad where haematinics were prescribed to maintain her $\mathrm{Hb}$ above $10 \mathrm{gm} \%$.

She was admitted at hospital electively at 34 completed weeks of gestation. On admission she received two doses of Inj Betamethasone 24 hours apart for fetal lung maturity. Prior to surgery all the blood components were reserved.

On the day of surgery, a multidisciplinary approach was adopted. A protocol was drafted and familiarised to the team members who consisted of senior obstetricians, senior anaesthesiologist, senior surgeon, and senior OT staff.

Massive transfusion protocol was triggered.

On opening the abdomen, uterus was visualised with hyper vascular lower segment with no normal myometrium, only the serosa was intact seen as a bulge in the lower uterine segment.

A classical caesarean section with transverse incision was performed and healthy $2.6 \mathrm{~kg}$ female baby was delivered by breech. The uterine incision was closed with placenta in situ.

The myometrium was found completely invaded by placenta leaving no choice but to proceed with obstetric hysterectomy. Prophylactic bilateral internal iliac artery ligation was done to prevent enormous haemorrhage during surgery which could compromise patient's life. During Obstetric hysterectomy, intentional cystectomy was done as posterior wall of bladder was strongly adherent to the uterus, which was repaired in 2 layers followed by putting a suprapubic catheter. An omental graft was kept between the bladder base and vaginal vault for avoiding the risk of VVF. She received in all 6 units of PRBCs, 6 units of FFP and 1 unit of SDP pre and intraoperatively, and 1 unit PRBC postoperatively.

Postoperative period was uneventful. Patient received antibiotic Inj. Cephotaxime. On day 10 stitches were removed, wound was healthy. Suprapubic catheter was removed on day 21 after doing micturating cystourethrogram. Both mother and baby were healthy at discharge. They are presently doing well.

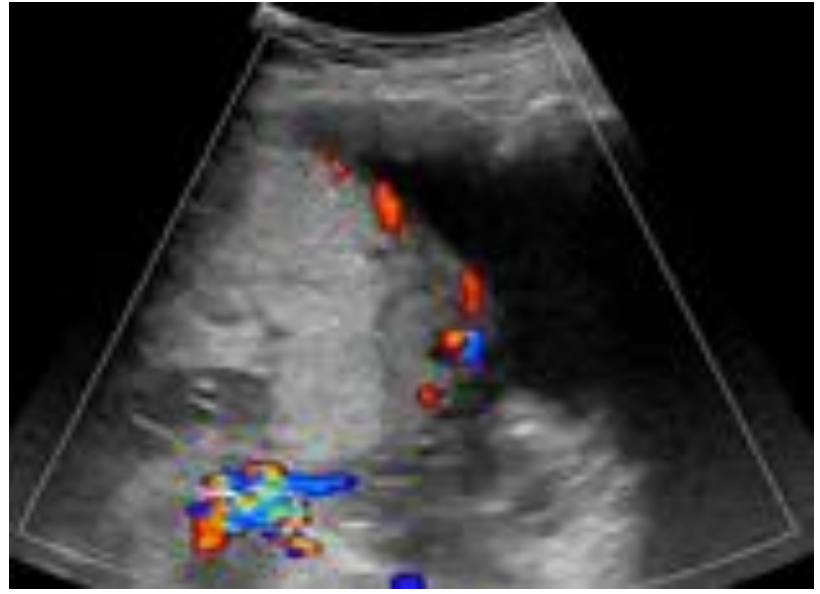

Figure 1: Ultrasonic picture at 34 weeks of gestation age showing invasion of blood vessels from uterus to posterior wall of bladder.

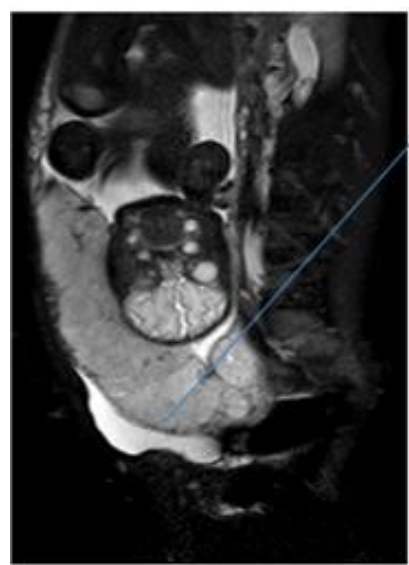

Loss of clear zone between the anterior wall of uterus and posterior wall of bladder called as 'bladder line' suggestive of placenta percreta.

Figure 2: MRI picture of case at 34 weeks of gestation age showing loss of clear plane between the uterus and posterior wall of bladder suggestive of placenta percreta.

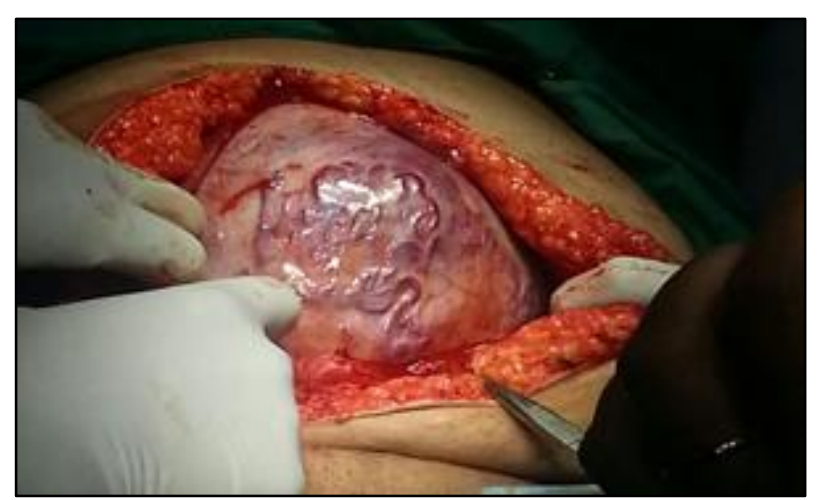

Figure 3: Hyper vascular lower uterine segment.

\section{DISCUSSION}

Placenta accreta is a life threatening condition which causes massive haemorrhage necessitating Obstetric hysterectomy in majority cases. Previous caesarean 
deliveries accentuate the incidence of placenta accreta greatly. $^{3}$ Majority of times the woman remains asymptomatic as was in our case and detected accidentally during surgery or on antenatal ultrasound but a classical case presents it with painful vaginal bleeding, gross haematuria, and sometimes dull continuous pain in lower abdomen. With gray-scale ultrasonography and MRI techniques, the condition can be diagnosed as early as 10 weeks. ${ }^{4}$ When a woman has history of risk factors for adherent placenta, high level of suspicion should be kept and every attempt should be made to diagnose placenta percreta/accrete antenatally.

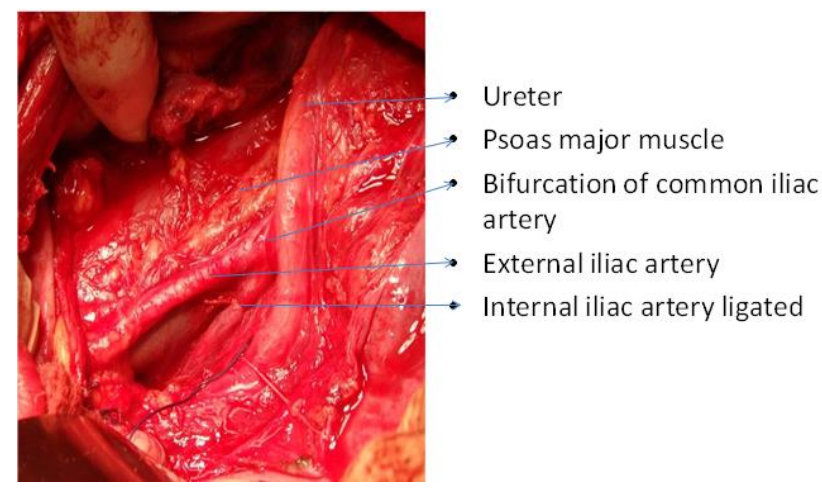

Figure 4: Internal iliac artery ligation.

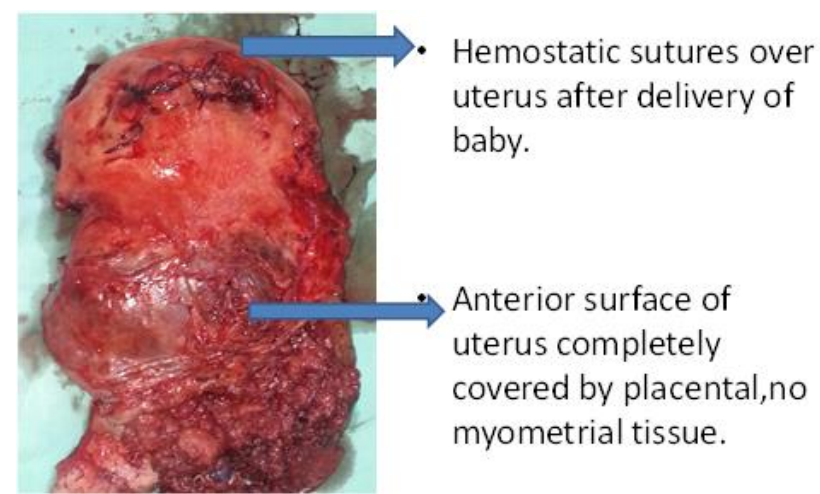

Figure 5: Post-operative specimen.

From management point of view, choice of treatment has to be carefully individualised. Uterine conservation may be considered when the woman very much wants to preserve her fertility. She should be counselled extensively regarding the risks of hemorrhage, infection, possible need for intra or postoperative lifesaving hysterectomy, and suboptimal outcomes in future pregnancies. Uterine conservation is also considered when hysterectomy is thought to have an unacceptably high risk of hemorrhage or injury to other organs, which may be mitigated by leaving the placenta in situ. ${ }^{5}$

One alternative approach, Triple $\mathrm{P}$ procedure was described by Chandraharan $\mathrm{E}$ and co-workers as a conservative surgical alternative to peripartum hysterectomy for placenta accreta that entails perioperative placental localization, pelvic devascularisation, placental non-separation with myometrial excision. ${ }^{6}$

In our case, we preferred planned caesarean hysterectomy with placenta in situ over conservative technique as future fertility was not desired.We triggered massive transfusion protocol and performed bilateral internal iliac artery ligation prophylactically so as to replace and reduce enormous amount of blood loss. And the positive and encouraging outcome in this case adds to the claim that surgical management avoids risks of delayed and emergency hysterectomies.

Management by a multidisciplinary team and delivery in a tertiary care facility improves outcome and lower complication rates.

Many studies reported to avoid internal iliac (hypogastric) artery ligation because it is time consuming, operator dependent, ineffective (without hysterectomy) for controlling pelvic hemorrhage in up to 60 percent of cases and precludes use of selective pelvic angiography and embolization if needed subsequently. ${ }^{7-9}$ We have done prophylactic internal iliac ligation by anterior approach through broad ligament and found it to be less time consuming, less operator dependent (as less amount of retraction is required) and found to be complimentary to caesarean hysterectomy in reducing the amount of blood loss.

Placenta percreta with bladder invasion may require partial cystectomy. In one review of 54 cases of placenta percreta invading the bladder, partial cystectomy was performed in 24 of the 54 patients. ${ }^{10}$ Ideally, a urogynecologist, urologist, or gynecologic oncologist should be consulted when the bladder is involved. Cystoscopy or intentional cystotomy at surgery may be helpful for assessing the degree of bladder, and possible ureteral, involvement. ${ }^{11,12}$ We also had to do intentional cystostomy in this case. Regardless of the decision whether to remove the bladder, anterior bladder wall cystotomy is particularly helpful for defining dissection planes and determining whether posterior bladder wall resection is required. $^{13,14}$

\section{CONCLUSION}

Counselling and multidisciplinary team involvement have shown strong success rate in treatment for placenta percreta. Early planning can ensure optimal outcomes for woman. Counselling allows for women's autonomy in making decisions such as possible termination of the pregnancy, early delivery via caesarean section, or planned hysterectomy. Triggering massive transfusion protocol promptly will prevent morbidity by reducing haemorrhage. Performing measures like internal iliac artery ligation, uterine artery embolization helps to reduce blood loss during surgery. 
Funding: No funding sources

Conflict of interest: None declared

Ethical approval: Not required

\section{REFERENCES}

1. Tantbirojn P, Crum CP, Parast MM. Pathophysiology of placenta percreta: the role of decidua and extravillous trophoblast. Placenta. 2008;29:63945 .

2. Wu S, Kocherginsky M, Hibbard JU. Abnormal placentation: twenty-year analysis. American Journal of Obstetrics and Gynecology. 2005;192(5):1458-61.

3. Clark SL, Koonings PP, Phelan JP. Placenta previa/accreta and prior cesarean section. Obstet Gynecol. 1985;66:89-92.

4. Sze-Yan C, Chong-Pun C. The sonographic appearance and obstetric management of placenta accrete. International Journal of Women's Health. 2012;2(4):587.

5. Fox KA, Shamshirsaz AA, Carusi D. Conservative management of morbidly adherent placenta: expert review. Am J Obstet Gynecol. 2015;213:755.

6. Chandraharan E, Rao S, Belli AM, Arulkumaran S. The Triple-p procedure as a conservative surgical alternative to peripartum hysterectomy for placenta percreta. Int J Gynaecol obstet. 2012;117(2):191-4.
7. Clark SL, Phelan JP, Yeh SY. Hypogastric artery ligation for obstetric hemorrhage. Obstet Gynecol. 1985;66:353.

8. Papp Z, Tóth-Pál E, Papp C. Hypogastric artery ligation for intractable pelvic hemorrhage. Int $\mathbf{J}$ Gynaecol Obstet. 2006;92:27.

9. Unal O, Kars B, Buyukbayrak EE. The effectiveness of bilateral hypogastric artery ligation for obstetric hemorrhage in three different underlying conditions and its impact on future fertility. J Matern Fetal Neonatal Med. 2011;24:1273.

10. Washecka R, Behling A. Urologic complications of placenta percreta invading the urinary bladder: a case report and review of the literature. Hawaii Med J. 2002;61:66.

11. Eller AG, Porter TF, Soisson P, Silver RM. Optimal management strategies for placenta accreta. BJOG. 2009;116(5):648-54.

12. Bakri YN, Sundin T. Cystotomy for placenta previa percreta with bladder invasion. Urology. 1992;40:580.

13. Abbas F, Talati J, Wasti S. Placenta percreta with bladder invasion as a cause of life threatening hemorrhage. J Urol. 2000;164:1270-4.

14. Pedicled omental graft in repair of large ,difficult vesicovaginal fistulae. Int $\mathrm{J}$ Gynaecol Obstet. 1980;17(6):556-9

Cite this article as: Gadappa S, Deshpande S, Rajpurohit K, Kalyankar B. Placenta percreta - an audacious experience. Int J Reprod Contracept Obstet Gynecol 2016;5:3261-4. 